NSF-ITP-92-100

TPI-MINN-92-32/T

June 1992

\title{
The Isgur-Wise function in the small velocity limit
}

\author{
B. Blok \\ Institute for Theoretical Physics \\ University of California at Santa Barbara \\ Santa Barbara, CA 93106 \\ and \\ M. Shifman \\ Theoretical Physics Institute \\ University of Minnesota \\ Minnesota, MN 55455
}

\begin{abstract}
We discuss the Isgur-Wise function $\xi(y)$ in the small velocity (SV) limit within the QCD sum rule method. The behavior of $\xi(y)$ in the SV limit is sensitive to the particular form of the duality relations used to decontaminate the sum rule predictions from the continuum contribution. Peculiarities of the duality relations in the problem at hand are revealed. It is shown that the proper requirements of duality and angular isotropy for $\mathrm{S}$ wave states lead to an unambiguous form of the sum rules for the Isgur-Wise function. We illustrate the constraints due to these requirements using a toy model of the harmonic oscillator. The slope parameter and the shape of $\xi(y)$ are determined.
\end{abstract}




\section{INTRODUCTION.}

Strong interactions in the quark systems become simpler if one of the quarks considered is infinitely heavy. Then its motion is effectively classic and can be considered as given in the sense that the surrounding gluons and light quarks (we will refer to this component as to the light cloud, which is equivalent to more commonly used notion of "brown muck") do not affect it at all. The fact that the simplest hadronic system one can invent is built from an infinitely heavy quark plus the light cloud has been noted long ago . ${ }^{1}$ The revival of interest to such systems we are witnessing now is due to explicit introduction of new symmetries ${ }^{2}$ taking place if there are several heavy quarks, and $m_{Q} \gg \Lambda_{\mathrm{QCD}}$ where $m_{Q}$ is the heavy quark mass. The initial impetus in this direction has been given by the work 3 where the $B \rightarrow D$ transition has been analysed in the so called small velocity (SV) limit, see below. The culminating point in the recent achievements in the "heavy" quark-"light" quark systems is the formulation of the Heavy Quark Effective Theory ${ }^{4}$ (for a review see Ref. 5), which ideally suits for describing regularities of this sector of the hadron world.

In the semileptonic transitions of heavy mesons of different flavors the heavy quark symmetry is reflected in the universal Isgur-Wise (IW) function. Assume we are interested in the current induced transitions (it can be either vector $V_{\mu}$ or axial $A_{\mu}$ current) between two heavy mesons, $M_{i}$ and $M_{f}$. The quark content of $M_{i, f}$ is $Q_{i, f} \bar{q}$ where $Q_{i}\left(Q_{f}\right)$ is the initial (final) heavy quark and $\bar{q}$ is a light antiquark. For instance, we can take b as $Q_{i}$ and c as $Q_{f}$. Moreover, assume for definiteness that $M_{i}$ is the ground state pseudoscalar (generic B). $M_{f}$ can be either the ground state pseudoscalar or the ground state vector (generic $D$ or $D^{*}$ ). In general, the set of transition matrix elements of $V_{\mu}$ and $A_{\mu}$ involves a large number of apriori unrelated formfactors. In the limit $m_{Q} \rightarrow \infty$ all these matrix elements are described by a single function which can be introduced in the following way:

$$
<P_{Q_{f} \bar{q}}(v)\left|V^{\mu}\right| P_{Q_{i} \bar{q}}\left(v_{i}\right)>=\sqrt{M_{f} M_{i}} \xi(y)\left(v_{f}^{\mu}+v_{i}^{\mu}\right) .
$$

Here $P_{Q \bar{q}}$ stands for the pseudoscalar meson with the quark content $Q \bar{q}, V^{\mu}=\bar{Q}_{f} \gamma_{\mu} Q_{i}$, $M_{f}\left(M_{i}\right)$ denotes the mass of the final (initial) meson, $v^{\mu}$ is the four-velocity,

$$
v_{i, f}^{\mu}=P_{i, f}^{\mu} / M_{i, f},
$$

and, finally, the variable $y \equiv v_{f} v_{i}$. In the limit $m_{Q} \rightarrow \infty$ we stick to the quark and meson masses coincide. The peculiarity of the IW formfactor $\xi$ is that it depends only on the 
velocity transfer ( masses are irrelevant) ${ }^{2}$. If in the rest frame of the initial meson the final meson is also at rest then $\xi$ is trivially known ${ }^{3}$ :

$$
\xi(y=1)=1 .
$$

The normalization property of the IW function in the SV limit is essentially not dynamical, it merely expresses the symmetry of the strong interaction. At the same time the shape of the function $\xi(y)$ encodes dynamical properties of Quantum Chromodynamics. One can show that $\xi(y)$ is related to the Wilson line operator for specific contours and is, thus, one of the most fundamental objects in QCD. Dynamical QCD-based calculations of the IW function are, clearly, of general interest.

Several recent works are devoted to this issue. In Ref. 6,7,8,9 the IW function is treated within the QCD sum rule approach ${ }^{10}$, with results that seem to be quite promising. (The first applications of the QCD sum rules to the form factor problems are worked out in Ref. 11,12,13,14). Still the question is far from being closed since some aspects obviously require further study. One of the most evident examples is the small velocity (SV) limit ${ }^{3}$ of the IW function. The slope of the function $\xi$ at $y \rightarrow 1$ reflects an interesting characteristics of the formfactor (see below). In Ref. 7 it comes out infinite, an unphysical feature of the corresponding analysis. A remedy suggested in Ref. 8 eliminates the spurious infinity, but leaves the prediction for slope parameter rather uncertain, to say nothing about an apparent theoretical ambiguity contained in a prescription used in Ref. 8 which calls for clarification.

The present paper is specifically devoted to the analyses of the IW function in the SV limit. First, we discuss different specific aspects of the QCD sum rule calculations, elucidating new elements which go beyond the standard procedure ${ }^{10}$. The main focus is on the issue of how duality must be applied to estimate continuum contribution in the formfactor situation. In the SV limit at least one of the standard prescriptions is shown to be contradictory and the procedure of the continuum decontamination should be carried out in a specific way. Second, we comment on the additional information stemming from the Bjorken sum rule ${ }^{15}$ which relates the slope of $\xi$ in the SV limit to nondiagonal formfactors of the type $\left.\left.Q_{i} \bar{q}\right|_{0^{-}} \rightarrow Q_{f} \bar{q}\right|_{1^{+}}$

Our main results can be summarized as follows. The duality for the three-point functions must be understood not in a local, but in a generalized sense, as duality between integrated spectral densities. This is explained in detail in section 2 on the example of a toy model of the harmonic oscillator. The sum rules for the Isgur-Wise function and its slope parameter $\rho^{2}$ are described in section 3. We argue that the symmetry requirements and the proper duality 
lead to an unambiguous form of the sum rules. This, in turn, leads to the elimination of uncertainties noticed in Ref. 8,9. In section 4 we analyse the sum rules and get predictions for the Isgur-Wise function at small $y$ and for $\rho^{2}$. We also show the self-consistency of our approach using the Bjorken sum rule.

\section{CALIBRATING THE SUM RULE APPROACH IN THE HARMONIC OSCILLATOR.}

In this section we shall test various elements of the method ${ }^{10}$ in peculiar kinematics inherent to the problem at hand. To this end a toy model is considered where there are no gluons and the interaction between $\mathrm{q}$ and $\mathrm{Q}$ is pure potential. For simplicity we will choose the harmonic oscillator potential,

$$
V(\vec{r})=\frac{m \omega^{2} \vec{r}^{2}}{2}
$$

where $\vec{r}$ is the distance between $\mathrm{Q}$ and q, $\mathrm{m}$ is a mass of the "light quark".

It is convenient to work in the Breit reference frame (Fig.1). Before the photon emission the heavy quark force center moves with the velocity $\vec{v}$, after the emission its velocity is $-\vec{v}$, so that the momentum transfer $\mathrm{q}=(0, \vec{q})$, where $\vec{q}=2 M \vec{v}, M \rightarrow \infty$ is a mass of the heavy quark. Since our toy model is nonrelativistic (with respect to the light quark q) we have to assume that $\omega \ll m$ and, moreover, $|\vec{v}| \ll \sqrt{\omega / m}$. The latter constraint is imposed because we are interested in the SV limit. In other words the formfactor is expanded in powers of $\vec{v}$, and only the terms $O\left(\vec{v}^{0}\right)$ and $O\left(\vec{v}^{2}\right)$ are kept. The first term trivially reduces to unity -an analog of eq. (3)-while the coefficient in front of $\vec{v}^{2}$ is the central object of our analysis.

Needless to say that in the potential model the diagonal formfactor can be found explicitly and in the straightforward way, as well as nondiagonal transitions involved in the Bjorken sum rule. The nonrelativistic analogue of the IW function has the form (in the Breit system)

$$
\xi\left(\vec{v}^{2}\right)_{\text {n.r. }}=\int d^{3} x \psi_{0}^{*}(\vec{r}) e^{-2 i m \vec{v} \vec{v}_{1}} \psi_{0}(\vec{r})
$$

Here $\psi_{0}$ is the ground state wave function and and the subscript "n.r." stands for "nonrelativistic". Eq. (5) implies that for the oscillator potential (4)

$$
\xi_{\text {n.r. }}=1-\frac{m}{\omega} \vec{v}^{2}+\ldots
$$


In order to make contact with the standard parametrization accepted in the relativistic case (see e.g. $\left.{ }^{16}\right)$,

$$
\begin{aligned}
& \xi(y)=1-\rho^{2}(y-1)+O\left((y-1)^{2}\right), \\
& y=v_{\mu}^{\prime} v^{\mu}
\end{aligned}
$$

it is convenient to present eq. (6) as

$$
\rho_{n . r .}^{2}=\frac{m}{2 \omega}
$$

Here the fact that

$$
y-1=2 \vec{v}^{2}+O(\vec{v})^{4}
$$

is explicitly taken into account. Notice that for genuinely non-relativistic systems parameter $\rho^{2}$ is large: $\frac{m}{\omega} \gg 1$.

Along with the diagonal formfactor (5) one can consider nondiagonal transition formfactors from $\mathrm{S}$ to $\mathrm{P}$-wave states, nonrelativistic analogues of the universal functions introduced in Ref. 16. In this case

$$
2 i v_{j} \tau_{\mathrm{n} . \mathrm{r}}=\int d^{3} x \psi_{0}^{*}(x) e^{-2 i m \vec{v} \vec{r}_{j}} \psi_{j}(x)
$$

where $\psi_{j}(x) \quad(j=1,2,3)$ is the three-component wave function of the P-wave state:

$$
\psi_{j}=\sqrt{\frac{3}{4 \pi}} \frac{r_{j}}{|r|} R_{1}(r)
$$

The explicit forms of $\psi_{0}$ and $\psi_{j}$ can be found in the standard textbooks( see e.g. Ref. 17). At small $\vec{v}^{2}$ the transition amplitude is proportional to $\vec{v}$. In the general case there are transitions from the ground state $\psi_{0}$ to all excited P-wave states. For the harmonic oscillator, however, the only nonvanishing $\tau$ is that due to the transition to the lowest P-wave state.

Now, the Bjorken sum rule ${ }^{15}$ takes the form:

$$
\rho_{n . r .}^{2}=r_{\text {n.r. }}^{2}\left(\vec{v}^{2}=0\right)
$$

The latter equation expresses the completeness of the full set of the wave functions. From eq. (10) it trivially stems that

$$
\tau\left(\vec{v}^{2}=0\right)=-\sqrt{\frac{m}{\omega}} \frac{1}{\sqrt{2}}
$$

Comparing eqs. (8), (12) and (13) we see that the Bjorken sum rule is satisfied. Of course, there is no doubt that it should be satisfied. We do this simple exercise here only because we 
want to set the framework and test the sum rule approach, to be applied in more complex environment of quarks and gluons.

Let us pretend now that the wave functions are unknown, and the only quantities we are able to calculate reliably are the correlation functions at short (euclidean) times. Specifically, we consider

$$
\begin{aligned}
S\left(\vec{v} ; \tau_{1}, \tau_{2}\right) & =\int d^{3} r K\left(0, \tau_{1}+\tau_{2} \mid \vec{r}, \tau_{1}\right) e^{-2 i m \vec{v} \vec{r}} K\left(\vec{r}, \tau_{1} \mid 0,0\right) \\
& \equiv \int d^{3} r \Sigma_{l} e^{-E_{l} \tau_{2}} \psi_{l}(0) \psi_{l}^{*}(\vec{r}) e^{-i m \vec{v} \vec{r}} \Sigma_{n} \psi_{n}^{*}(0) \psi_{n}(\vec{r}) e^{-i m \vec{v} \vec{r}} e^{-E_{n} \tau_{1}}
\end{aligned}
$$

This is the nonrelativistic analog of three point function. Here $K(\vec{r}, \tau \mid \overrightarrow{0}, 0)$ is the amplitude (time-dependent Green function ) of the propagation from the point $(0,0)$ to the point $\vec{r}, \tau$ in the Euclidean time. If $\tau_{1,2} \rightarrow \infty$ only the ground state contributes and $S\left(\vec{v}, \tau_{1}, \tau_{2}\right) \rightarrow$ $e^{-E_{0} T}\left|\psi_{0}(0)\right|^{2} \xi_{\text {n.r. }}\left(\vec{v}^{2}\right), \quad T=\tau_{1}+\tau_{2}$. For the harmonic oscillator $K$ is known exactly for all $\tau$ (see e.g. Ref. 18),

$$
K(\vec{x}, \tau \mid 0,0)=\left(\frac{m \omega}{2 \pi \sinh \omega \tau}\right)^{3 / 2} e^{-\frac{m \omega}{2 \sinh (\omega \tau)} \vec{x}^{2} \cosh (\omega \tau)} .
$$

However we shall base our estimates on the short time expansion of $\mathrm{K}$ in order to imitate QCD calculations, and then compare the results obtained in this way with the exact ones.

Integration over $\vec{r}$ in eq. (14) is readily carried out, and we arrive at the following $\vec{v}^{2}$ expansion

$$
S\left(\vec{v} ; \tau_{1}, \tau_{2}\right)=S_{0}+S_{1}+\ldots
$$

where $S_{i}$ is the term of the $i-t h$ order in $\vec{v}^{2}$,

$$
\begin{gathered}
S_{0}=\left(\frac{m \omega}{2 \pi}\right)^{3 / 2} \frac{1}{(\sinh \omega T)^{3 / 2}}, \quad T=\tau_{1}+\tau_{2} \\
S_{1}=\left(\frac{m \omega}{2 \pi}\right)^{3 / 2} \frac{1}{(\sinh \omega T)^{3 / 2}} \frac{\sinh \left(\omega \tau_{1}\right) \sinh \left(\omega \tau_{2}\right)}{\sinh \omega T} \frac{m}{\omega}\left(-2 \vec{v}^{2}\right) .
\end{gathered}
$$

Notice that the zero-order term is just the same as for two-point functions (see e.g. Ref. 19), a straightforward consequence of the normalization theorem ${ }^{3}$. It is well known that the sum rule approach gives beautiful results for the positions of the S-wave levels in Quantum mechanics ${ }^{20}$, a refined expression of duality which works in a very transparent way in this case. We will concentrate on the term $O\left(\vec{v}^{2}\right)$ determining the slope of the formfactor.

Combining eqs. (18) and (17) in the limit $\tau_{1,2} \rightarrow \infty$ one would recover eq. (8) ( e.g. by considering the ratio $S_{1} / S_{0}$.) We would like to examine how far one can go with the short $\tau$ expansion. 
Keeping only the first terms of the expansion already produces a reasonable estimate even disregarding the contribution due to higher states ( the latter is at the center of the discussion below). Indeed, in the symmetric point $\tau_{1}=\tau_{2}=T / 2$

$$
S_{1} / S_{0}=\left(-2 \vec{v}^{2}\right) \frac{m}{\omega} \frac{x}{4}\left(1-\frac{1}{12} x^{2}+\ldots\right), \quad x \equiv \omega T .
$$

It is easy to see that the stability plateau is around $x \sim 2$; the corresponding estimate for $\rho^{2}$ is $\rho^{2} \sim \frac{m}{2 \omega}(2 / 3)$ (cf. eq. (8)). At this point the power correction constitutes $1 / 3$ of the leading term. Moreover, with 3 power terms included $\rho^{2} \sim(m /(2 \omega)) 0.8$. Agreement with the exact result (8) is evident, although not very impressive, of course, which is natural, since in the above analysis we completely ignored contamination due to higher states.

Let us discuss now how the higher states affect the analysis. $S_{0}$ is contributed by the S-levels only, situated at $E_{n}=\frac{3}{2} \omega+2 n \omega$, only diagonal transitions take place,

$$
S_{0}=\sum_{n=0}^{\infty}\left|\psi_{n}(0)\right|^{2} e^{-E_{n} T}
$$

where

$$
\begin{aligned}
\left|\psi_{n}(0)\right|^{2} & =\left(\frac{m \omega}{\pi}\right)^{3 / 2} \frac{(2 n+1) ! !}{2^{n} n !} \\
& \rightarrow \sqrt{\frac{E_{n}}{\omega}}\left(\frac{m \omega}{\pi}\right)^{3 / 2} \sqrt{\frac{2}{\pi}} \text { at } E_{n} \gg \omega .
\end{aligned}
$$

Eq. (21) perfectly matches the "bare" spectral density, i.e. that determining $S_{0}$ in the limit when the interaction is switched off, $\omega \rightarrow 0$,

$$
\sigma_{0}^{\text {bare }}=\frac{1}{\sqrt{2 \pi}}\left(\frac{m}{\pi}\right)^{3 / 2} \sqrt{E}, S_{0}^{\text {bare }}=\int_{0}^{\infty} \sigma_{0}^{\text {bare }} e^{-E T} d E .
$$

Indeed, the smeared sum of delta-functions, $\sigma=\sum_{n} \sqrt{\frac{2}{\pi}} \sqrt{\frac{E_{n}}{\omega}}\left(\frac{m \omega}{\pi}\right)^{3 / 2} \delta\left(E-E_{n}\right)$, coincides with eq. (22). The standard step-like model ${ }^{10}$ with $\sigma_{0}$ from eq. (22) and the threshold energy $E_{0}=\frac{5}{2} \omega$ gives excellent approximation for higher states ${ }^{20}$.

Let us proceed to less studied case of $S_{1}$ where, unlike $S_{0}$, we do encounter peculiarities of the form-factor situation. From the large $\tau$ expansion of eq. (18) we learn that $S_{1}$ contains both the diagonal and off-diagonal transitions,

$$
\begin{aligned}
S_{1 \text { diag }} & =\sum_{k=0}^{\infty}\left(\frac{-2}{3} m^{2} \vec{v}^{2}\right)\left(r_{k, k}^{2}\right)\left|\psi_{k}(0)\right|^{2} e^{-E_{k} T} \\
& =\left(-2 \vec{v}^{2}\right)\left(\frac{m}{\omega}\right)\left(\frac{m \omega}{2 \pi}\right)^{3 / 2}\left(\frac{1}{\sinh \omega T}\right)^{5 / 2} \frac{1}{2} \cosh \omega T
\end{aligned},
$$


and

$$
\begin{aligned}
S_{1 o f f-\text { diag. }} & =\sum_{k=0}^{\infty}\left(-\frac{2}{3} m^{2} \vec{v}^{2}\right)\left(r^{2}\right)_{k, k+1} \psi_{k}(0) \psi_{k+1}^{*}(0) e^{-E_{k} T}\left(e^{-2 \omega \tau_{2}}+e^{-2 \omega \tau_{1}}\right) \\
& =\left(-2 \vec{v}^{2}\right)\left(\frac{m}{\omega}\right)\left(\frac{m \omega}{2 \pi}\right)^{3 / 2} \frac{1}{(\sinh \omega T)^{5 / 2}}(-1 / 4) e^{\omega T}\left(e^{-2 \omega \tau_{2}}+e^{-2 \omega \tau_{1}}\right) .
\end{aligned}
$$

Here

$$
r_{l, k}^{2}=\int d^{3} r \psi_{l}^{*}(\vec{r}) \vec{r}^{2} \psi_{k}(\vec{r})
$$

and we have used the fact specific for the oscillator that the off-diagonal transitions are only from $l$ to $l \pm 1 \mathrm{~S}$ wave state. Thus, the ground state is connected only to the first excited S-wave state. $\psi_{k}(\vec{r})$ stands for the wave function of the $\mathrm{k}$-th $\mathrm{S}$-wave state. The set of points where the physical spectral density is concentrated is presented in Fig. 2.

Now, one can easily convince oneself that the residue of the $\mathrm{k}$-th state contributing to $S_{1 \text { diag }}\left(E_{k}=(3 / 2) \omega+2 \omega k\right)$ is equal to

$$
\left(-\frac{2}{3} m^{2} \vec{v}^{2}\right)\left(r^{2}\right)_{k, k}\left|\psi_{k}(0)\right|^{2}=-2 \vec{v}^{2}\left(\frac{m}{2 \omega}\right)\left(\frac{m \omega}{\pi}\right)^{3 / 2}\left\{\frac{(4 k+3)}{3} \frac{1}{2^{2 k}} \frac{1}{(k !)^{2}}(2 k+1) !\right\} .
$$

Furthermore, for the off-diagonal transitions, eq. (24), the residues are

$$
\left(-\frac{2}{3} m^{2} \vec{v}^{2}\right)\left(r^{2}\right)_{k, k+1} \psi_{k}(0) \psi_{k+1}^{*}(0)=-2 \vec{v}^{2}\left(\frac{m}{2 \omega}\right)\left(\frac{m \omega}{\pi}\right)^{3 / 2}(-1)\left\{\frac{2 k+3}{3} \frac{1}{(k !)^{2}} \frac{(2 k+1) !}{2^{2 k}}\right\} .
$$

Notice the important minus sign appearing in passing from eq. (26) to eq. (27) .

At this point it becomes clear that the standard picture of local duality we got used to in the case of the sum rules for the two-point functions ${ }^{10}$ (see e.g. Ref. 21 ) fails in the formfactor situation, at least in the kinematics at hand (expansion of the formfactor near the SV limit). Indeed, the standard picture would imply that a few individual levels smeared over a natural duality domain produce (locally) the same spectral density inside this domain as the one obtained from free motion. We will see shortly that a conventional averaging of eqs. (26) and (27) leads to something quite different from the "bare" spectral density, even in sign.

First we need to calculate the function $S\left(\vec{v} ; \tau_{1}, \tau_{2}\right)$ defined in eq. (14) for the case of free motion $(\omega=0)$. This is a straightforward exercise since in this case

$$
S_{\text {bare }}=\iint \frac{d^{3} \vec{p} d^{3} \vec{p}^{\prime}}{(2 \pi)^{6}} d^{3} \vec{r} e^{i \vec{p} \vec{r}-i \vec{p} \vec{r}} \exp \left\{-\frac{\vec{p}^{2}}{2 m} \tau_{1}-\frac{\vec{p}^{2}}{2 m} \tau_{2}\right\} ;
$$


$\vec{r}$ is the distance between the particle and the force center; $\frac{\vec{p}^{2}}{2 m}$ and $\frac{\vec{p}^{2}}{2 m}$ are energies.

We begin with a free particle with the momentum $\vec{p}^{\prime}+m \vec{v}$ in the Breit frame $\left(\vec{p}^{\prime}\right.$ is in the rest frame of the force center Q, see Fig.1), and end up with the particle with the momentum $\vec{p}-m \vec{v}$ in the Breit frame $(\vec{p}$ in the rest frame of the force center $\mathrm{Q}$, after it emits a quanta " $\gamma$ " which carries away $\vec{q}=2 M \vec{v}$, Fig.1). Since there is no interaction, nothing happens to the particle in the process of scattering of the force center,

$$
\vec{p}^{\prime}+m \vec{v}=\vec{p}-m \vec{v}
$$

Eq. (29), obvious as it is, also formally stems from eq. (28) which can be readily transformed to

$$
S_{\text {bare }}=\frac{1}{8 \pi^{2}} \frac{m}{|\vec{v}|} \int_{\Sigma} d E d E^{\prime} e^{-E \tau_{1}-E^{\prime} \tau_{2}}
$$

where the integration in the right-hand side runs over the domain $\Sigma$ depicted in Fig.3.

In the limit $v \ll 1$ we are interested in the bare spectral density

$$
\sigma_{\text {bare }}(E)=\frac{1}{8 \pi^{2}} \frac{m}{|\vec{v}|}
$$

is very large and is concentrated in the narrow strip near the diagonal $E=E^{\prime}$ (Fig.3). If, instead of $\mathrm{E}$ and $E^{\prime}$, one introduces the variables

$$
A=E^{\prime}-E, \quad B=\frac{1}{2}\left(E^{\prime}+E\right)
$$

the boundary of $\Sigma$ is given by the equation

$$
B=\frac{1}{8 m \vec{v}^{2}} A^{2}+\frac{1}{2} m \vec{v}^{2}
$$

Moreover, $\mathrm{B}$ varies in the interval $\left[m v^{2} / 2, \infty\right)$; for given $\mathrm{B}$ the maximal and minimal values of $\mathrm{A}$ are $\pm\left(B-\frac{m v^{2}}{2}\right)^{1 / 2}\left(8 m v^{2}\right)^{1 / 2}$. Thus, at $v \rightarrow 0$ the width of the shaded strip in Fig. 3it is proportional to $\mathrm{v}$ - vanishes. Naively, one would say that the bare spectral density is dual to diagonal transitions only (We hasten to add that the latter statement is not correct). The fact that $\sigma_{\text {bare }}>0$ will be important in what follows.

One more observation important for understanding how the duality can be implemented in the case at hand. The process with the interaction switched off which must be dual to that with the bound state propagation looks as follows. The propagation of the force center before and after scattering is like the cannon ball, (Fig.1) and we need not consider it. The propagation of the light "particle" is free. In the laboratory (Breit) frame we fix its energy, 
otherwise allowing it to propagate freely in any direction it wants from the point of emission to the point of absorbtion. Fixing the energy of the light particle in the Breit frame means that we fix

$$
B=\frac{1}{2}\left(E+E^{\prime}\right)=\mathcal{E}+\frac{m v^{2}}{2}
$$

where the energy in the Breit frame is denoted as $\mathcal{E}$. On the other hand, allowing for all possible angles for the vector $\vec{p}_{0}=\vec{p}^{\prime}+m \vec{v}=\vec{p}-m \vec{v}$ implies that $A=E-E^{\prime}$ must be allowed to take all kinematically possible values. Thus it is clear that the domains of duality can be only those depicted on Fig. 4. Any other orientation of the duality domains by no means can be acceptable, and as a matter of fact, leads to unphysical result for the slope parameter as will be discussed in more detail below.

Let us now return to the problem of duality and discuss it in more detail. Consider first the spectral density for the free particle. This density is concentrated in a small domain of the width $\sim|\vec{v}| \sqrt{m \mathcal{E}}$ around the diagonal and is positive. The Taylor coefficients of expansion of $S_{\text {bare }}$ in $v^{2}$ arise through expanding the boundary of the duality domain in $\vec{v}^{2}$ and expanding the integration variables. The duality domain should be chosen as a certain interval in $\mathcal{E}$. From eq. (33) we see that for fixed $\mathcal{E} A_{\max }$ and $A_{\min }$ have no $v^{2}$ expansion. The only $v^{2}$ dependence comes from eq. (34) when one expresses $\mathrm{B}$ in terms of $\mathcal{E}$ and $v^{2}$ and, then, expands in $v^{2}$. To illustrate this point it is instructive to consider $S_{\text {bare }}$ at $\tau_{1}=\tau_{2}=T / 2$. Eq. (30) implies then that

$$
S_{\text {bare }}\left(\tau_{1}=\tau_{2}=T / 2\right)=\frac{1}{8 \pi^{2}} \frac{m}{v} \int_{0}^{\infty} d \mathcal{E} \int_{A_{\min }}^{A_{\max }} d A \exp -\left(\mathcal{E}+m v^{2} / 2\right) T .
$$

The width of the $A$ integration is $4 \sqrt{2 m \mathcal{E}} v$. (As has been already mentioned, this width scales as $\sim(m \omega)^{1 / 2} v \rightarrow 0$ at $\left.v \rightarrow 0\right)$. As a result,

$$
S_{\text {bare }}\left(\tau_{1}=\tau_{2}=\frac{T}{2}\right)=\frac{1}{\sqrt{2} \pi^{2}} m^{3 / 2} \int_{0}^{\infty} d \mathcal{E}^{1 / 2} \exp -\left(\mathcal{E}+m v^{2} / 2\right) T
$$

which coincides, of course, with the small $T$ limit of eqs. (17), (18), being expanded in $v^{2}$. The $v^{2}$ term in the expansion, we are most interested in, in particular, takes the form

$$
\begin{aligned}
S_{1} & =-m v^{2} T \frac{1}{2 \sqrt{2} \pi^{2}} m^{3 / 2} \int_{0}^{\infty} d \mathcal{E} \mathcal{E}^{1 / 2} \exp -\mathcal{E} T \\
& =-m v^{2} \frac{1}{4 \sqrt{2} \pi^{2}} m^{3 / 2} \int_{0}^{\infty} d \mathcal{E} \mathcal{E}^{-1 / 2} \exp -\mathcal{E} T
\end{aligned}
$$

The last line in this expression contains no explicit $T$ dependence except the exponential. 
On the other hand, consider the genuine spectral density for the oscillator. The spectral density for the Taylor coefficients in the expansion of $\mathrm{S}$ in powers of $v^{2 n}$ is spread over the length of $2 n \omega$ from the diagonal (Fig. 2). The reason is that it is proportional to matrix elements of $r^{2 n}$. For $\mathrm{n}=1$ we see that the spectral density is spread over the length $2 \omega$ as depicted in Fig.2. Consequently, we see that the spectral density for $S\left(\vec{v}^{2} ; \tau_{1}, \tau_{2}\right)$ is spread over the whole plane. The area in which it is concentrated has no correlation with the area where the free particle density is concentrated. The boundary of the area where the free particles spectral density is located is determined by $\mathcal{E}$ and $\vec{v}^{2}$, the analogous boundary for oscillator is infinite (or determined by $\omega$ for Taylor coefficients). Clearly, due to this reason the oscillator spectral density can not be locally dual to the spectral density for free particles in the usual sense. We conclude that the local duality between the spectral densities for the oscillator and for the free particles is absent in the formfactor situation considered here.

Although the local duality is absent, there still remains a weaker version of duality, namely a generalized duality. The oscillator spectral density integrated in the A-direction will be matched by the spectral density for the free particles integrated over the corresponding region, provided the weight function in the $\mathcal{E}$ direction is exponential.

Let us explain this assertion in more detail. Start with $S_{0}-$ the Taylor coefficient at zero order in the expansion of $\mathrm{S}$ in powers of $\vec{v}^{2}$. For the oscillator this density is proportional to $\sqrt{\mathcal{E}}$ and is concentrated on the diagonal. For free particles this is a constant spread over a narrow area depicted in Fig.4. Let us now integrate the free particle spectral density between the boundaries in the direction orthogonal to the diagonal (here and below we shall call this direction the A-direction). We immediately get the spectral density $\sim \sqrt{\mathcal{E}}$. We conclude that in this case the oscillator density is dual to free particle density integrated over the permitted interval in A direction. Note that the latter coincides with the free particle density from the sum rules for the 2-point function (see eq. (23)). We thus find the origin of the duality for $S_{0}$ discussed above (see eqs. (19)-(23), where we discussed the duality for $S_{0}$ ). Already for $S_{0}$ the duality is, rigorously speaking, a generalized one.

Consider now $S_{1}$. We have already discussed above the bare spectral density. The integral representation for $S_{1}$ has the integrand $\sim v^{-1}$ and a very narrow integration domain, whose width in the $\mathrm{A}$ direction is $\sim v$. One can introduce an effective spectral density in the $B(\mathcal{E})$ direction performing explicitly integration over $A$. Then the singularity in $v$ is eliminated and the correct analytic properties in $v^{2}$ are realised in a trivial way. One can read off the relevant expression for the effective spectral density from eq. (36) .

Now, proceed to the analysis of $S_{1}$ from the other side, with the interaction switched 
on, $\omega \neq 0$. In this case our starting point is eqs. $(23),(24),(26),(27)$. A sketch of the spectral density is given on Fig. 2 where the k-th energy level is given by $E_{k}=(3 / 2+2 k) \omega$. Our task is to check that the generalized duality still takes place: the appropriately defined sum over $\mathrm{k}$ matches eq. (36). In order to check duality we must go to the limit $\omega / E_{k} \rightarrow 0$ and check that in this case the expansion becomes that for the free particle. Here however we encounter a difficulty. The series (23), (24) converge absolutely only when $\omega T$ is kept finite. Otherwise we encounter alternating series for $S_{\text {diag }}$ and $S_{\text {non.-diag. with the terms }}$ increasing like $k^{3 / 2}$. If the contribution of the $\mathrm{k}$-th diagonal transition is positive that of the neighboring off-diagonal transitions is negative. Generally speaking, such sum depends on the way of summation. The regularization of the sum is provided by the exponential factor $\exp -E_{k} T$ in eqs. (23), (24) . This factor prompts us how to carry out the summation.

Let us consider the symmetric point $\tau_{1}=\tau_{2}=T / 2, \quad T \rightarrow 0 \quad(k$ large $)$. It is easy to see that the k-th state contributing to $S_{1 \operatorname{diag}}$ has the residue proportional to

$$
k^{3 / 2}+\alpha k^{1 / 2}+\beta k^{-1 / 2}+\ldots
$$

while that contributing to $S_{1 \text { off-diag }}$ has the residue

$$
k^{3 / 2}+\tilde{\alpha} k^{1 / 2}+\tilde{\beta} k^{-1 / 2}+\ldots .
$$

In terms of energies

$$
S_{1 \operatorname{diag}}\left(E_{k}\right) \sim \sum_{k}\left(2\left(\frac{E_{k}}{\omega}\right)^{3 / 2}+\alpha\left(\frac{E_{k}}{\omega}\right)^{1 / 2}+\ldots\right) \frac{1}{\sqrt{\omega}} \exp \left(-E_{k} T\right) .
$$

For the nondiagonal case we get in the same way:

$$
\begin{aligned}
S_{1 \text { non.-diag. }}\left(E_{k}\right) \sim & -\sum_{k}\left(2\left(\frac{E_{k}}{\omega}\right)^{3 / 2}+\tilde{\alpha}\left(\frac{E_{k}}{\omega}\right)^{1 / 2}+\tilde{\beta}\left(\frac{\omega}{E_{k}}\right)^{1 / 2}+\ldots\right) \exp \left(-E_{k} T\right) \\
& \frac{1}{\sqrt{\omega}}\left(\exp \left(-2 \omega \tau_{1}\right)+\exp \left(-2 \omega \tau_{2}\right)\right) .
\end{aligned}
$$

Here $\alpha, \beta, \tilde{\alpha}, \tilde{\beta}$ are numerical constants. Notice that the weight factors in eqs. (39), (40) are different. It is clear that the dangerous terms in the expansion are those proportional to $\left(E_{k} / \omega\right)^{3 / 2},\left(E_{k} / \omega\right)^{1 / 2}$. Otherwise the series will smoothly go into (36) in the limit $\omega \rightarrow 0$.

Let us discuss the dangerous terms in more detail. Eqs. (23) , (24), (26), (27) can be combined in the following way:

$$
\begin{aligned}
& S_{1}=-2 v^{2}\left(\frac{m}{2 \omega}\right)\left(\frac{m \omega}{\pi}\right)^{3 / 2} e^{-(3 / 2)(\omega T)} \\
& \sum_{k=0}^{\infty} \frac{1}{2^{2 k}} \frac{1}{(k !)^{2}} \frac{(2 k+1) !}{3} e^{(-2 k \omega T)}\left\{(4 k+3)-(4 k+6) e^{-\omega T}\right\} .
\end{aligned}
$$


The parameter $T$ and characteristic values of $\mathrm{k}$ in the sum are not independent: the choice of $T$ dictates what values of $\mathrm{k}$ saturate the sum. The duality formally is to hold at large $\mathrm{k}$ (small $T, \quad T \sim k^{-1}+C k^{-2}+\ldots$ ). Then, one can expand the expression in braces in $T$,

$$
\begin{aligned}
\{\ldots\} & =(4 k+3)-(4 k+6)\left(1-\omega T+\frac{\omega^{2} T^{2}}{2}+\ldots\right) \\
& =-3+4 k \omega T+\ldots .
\end{aligned}
$$

Notice that in $O\left(T^{0}\right)$ the terms $\sim k$ cancel. This means that under the appropriate summation the terms $\sim\left(E_{k} / \omega\right)^{3 / 2}$ in eqs. (39) and (40) annihilate each other.

The next step is to inspect what happens at the next-to-leading order. Since the individual contributions grow like $k^{3 / 2}$ it is quite obvious that the sum is saturated by terms with $k=\frac{3}{4 T \omega}$ (one can see this from maximizing $k^{3 / 2} \exp (-2 k \omega T)$. As a result, -3 in eq. (42) is cancelled by $4 k \omega T$. In terms of eqs. (39) and (40) this fact implies cancellation of $O\left(\left(E_{k} / \omega\right)^{1 / 2}\right)$ parts.

As a result we are left with terms $O\left(\left(E_{k} / \omega\right)^{-1 / 2}\right)$. To see that the coefficient matches that in eq. (36) one must keep the term $T^{2}$ in eq. (42) as well to use a relation between $T$ and $k, T=\frac{3}{4 \omega k}+C k^{-2}$, truncated at $O\left(k^{-2}\right)$ level.

Notice that our way of summation of diagonal and nondiagonal parts precisely corresponds to the integration in the A direction. We conclude that the spectral density integrated in the A direction for the oscillator is dual to the spectral density for free particles, also integrated in the A direction. Notice that duality is valid not for local densities $\sigma\left(E, E^{\prime}\right)$, but only for integrated densities in the A direction $\sigma(\mathcal{E})$. It is also obvious, from the discussion above, that the integration in the A direction is the only way to ensure duality. Otherwise we encounter dangerous terms $\sim 1 / \omega$ and/or can even get terms with the wrong analytic properties ( not expandable in $\left.\vec{v}^{2}\right)^{7}$

These considerations directly affect the possible choice of the continuum model. It is clear that the considerations above lead to the choice of the continuum model depicted on Fig.4 as the only possibility .

We discussed above an analogue of the double Borel transformed sum rules. During the investigation of the 3-point functions some authors considered also the so called sum rules in the external field ${ }^{22,23,13,24}$, that correspond to single Borel transformation. A non-relativistic analogue of the single- Borel transformed QCD sum rules is the function

$$
S^{s i n}=\sum_{n}(S)_{n n}\left|\psi_{n}(0)\right|^{2} \exp -E_{n} T-\sum_{n, l} S_{n l} \frac{\exp \left(-E_{n} T\right)-\exp \left(-E_{l} T\right)}{T\left(E_{n}-E_{l}\right)} \psi_{n}(0) \psi_{l}^{*}(0) .
$$


Here

$$
S_{n l}=\int d^{3} \vec{r} \psi_{n}^{*}(\vec{r}) \exp (-2 i m \vec{v} \vec{r}) \psi_{l}(\vec{r})
$$

It is clear that this function differs from the function $\mathrm{S}$ given by eq. (14) (taken at $\tau_{1}=\tau_{2}$ ) by different weight for the nondiagonal transitions. The single Borel sum rules (we shall use this name instead of more lengthy single time Borel transformed sum rules) are widely used, e.g. in the study of the pion and proton formfactors $22,23,13,24$. This is the reason why we consider them here. It is easy to see that in the limit $T \rightarrow \infty$

$$
S^{\sin }\left(\vec{v}^{2}, T\right) \rightarrow\left(|\psi(0)|^{2} \xi_{\text {n.r. }}\left(\vec{v}^{2}\right)+\frac{\text { const }}{\omega T}\right) e^{-E_{0} T}
$$

Here const $=-\psi_{0}(0) \psi_{1}^{*}(0) S_{01}+\ldots$ measures the contribution of the nondiagonal transitions. Asymptotically at $T \rightarrow \infty$

$$
\omega T S^{\sin }\left(\vec{v}^{2}, T\right)=\left(a_{0} x+b\right) e^{-E_{0} T}
$$

where $x=\omega T$ and $a_{0}=|\psi(0)|^{2} \xi_{\text {n.r. }}\left(\vec{v}^{2}\right)$. From eqs. (23), (24) it is easy to find exactly the corresponding coefficient for the terms $\sim \vec{v}^{2}$ in $S^{\text {sin }}$ :

$$
S_{1}^{\sin }(T)=-2 \vec{v}^{2} \frac{m}{\omega}\left(\frac{m \omega}{2 \pi}\right)^{3 / 2} \frac{1}{(\sinh \omega T)^{5 / 2}} \frac{1}{2}\left(\cosh \omega T-\frac{\sinh \omega T}{\omega T}\right) .
$$

The r.h.s. of eq. (47) multiplied by $(\sinh (\omega T))^{3 / 2} \omega T$ is well approximated by a straight line (46) for $x=\omega T$ for sufficiently large $\mathrm{x}$. Indeed, it has the form

$$
\frac{S_{1}^{\sin \omega T}}{S_{0}}=\left(-2 v^{2}\right) \frac{m}{\omega} \frac{1}{2}(x \operatorname{coth}(x)-1) .
$$

Taking several first terms in the expansion around point $x=0$ we have:

$$
\frac{S_{1}^{\sin }}{S_{0}}=\left(-2 v^{2}\right) \frac{m}{\omega}\left(x^{2} / 6-\frac{1}{90} x^{4}+\ldots\right) .
$$

The r.h.s. of eq. (49) is well approximated by a straight line for $x \geq 2$. We get $\rho^{2} \sim \frac{m}{2 \omega} 0.7$. Taking 3 power correction terms we get $\rho^{2} \sim \frac{m}{2 \omega} 0.8$. This can be compared with eq. (19) and the discussion that followed. We see that in the case of the harmonic oscillator the single Borel sum rule works as good as double Borel sum rule.

We note however, that in the case of the electromagnetic formfactor of pion the single Borel sum rules give overestimated value of the formfactor and underestimated value of the slope parameter, although within the error bars (see Ref. 22,23). 
Consider now the duality for the single Borel sum rule, i.e. function $S^{s i n}$. $S_{1 \text { diag }}$ is the same as in eq. (23). The off-diagonal part is almost the same as in eq. (24), with $\tau_{1}=\tau_{2}=T / 2$. The only difference is that $e^{-2 \omega \tau_{1}}+e^{-2 \omega \tau_{2}}=2 e^{-\omega T}$ in this equation is substituted by $\left(1-e^{-2 \omega T}\right) /(\omega T)$ in eq. (47). These two expressions have identical first two terms in the $\omega T$ expansion. In accordance with the discussion above this means that the dangerous terms in $S^{s i n}$ are precisely the same as the dangerous terms in the double Borel sum rule considered above. The duality considerations are the same for both sum rules. When we write this sum rule in terms of the spectral density $\sigma(\mathcal{E})$ we do it after implicit integration in A direction.

Let us now summarize lessons that follow from the study of duality for three point functions for harmonic oscillator. Note that these lessons refer not only to the case at hand discussed in the remainder of this article, but also to other cases of the calculation of three-point functions in QCD in similar kinematics. First, considerations of duality and appropriate symmetries impose strict constraints on the continuum model which must correspond to the region of Fig. 4 Second, duality is not local. The duality in three- point functions is between integrated in the A direction spectral densities.

\section{SUM RULES FOR THE ISGUR-WISE FUNCTION.}

In this section we shall write the sum rules for the IW function and extend the analysis of the preceding section to the heavy quark case.

We begin from the double Borel sum rules for the IW function in the symmetric point $\sigma_{1}=\sigma_{2}=2 \sigma$, where $\sigma$ denotes the Borel parameter. This sum rule was already written in Ref. $6,7,8,9$. We refer the reader to these articles as well as to Ref. 1 for the general discussion of Borel transformed nonrelativistic sum rules in heavy quark systems and the derivation of the sum rule for the IW function. We have rechecked that the coefficients in front of condensate terms are correct. The bare spectral density can be obtained from calculations in Ref. 25 which were carried out for finite heavy quark masses. Using explicit expressions in that work it is not difficult to take the limit $m_{Q} \rightarrow \infty$. 
The sum rule for the IW function has the form:

$$
\begin{aligned}
& \frac{3}{8 \pi^{2}(y+1) \sqrt{y^{2}-1}} \iint_{\Sigma} d s_{1} d s_{2}\left(s_{1}+s_{2}\right) \exp -\frac{\left(s_{1}+s_{2}\right)}{2 \sigma} \\
& -a / 4+\frac{b(2 y+1)}{2^{6} 3 \sigma^{2}}=\frac{F^{2} \xi(y) \exp -E_{R} / \sigma}{4} .
\end{aligned}
$$

Here $a, b$ are the vacuum condensates averages:

$$
\begin{aligned}
& a=<\bar{\psi}(0) \psi(0)>_{0}=-(0.24)^{3} \quad \mathrm{GeV}^{3} \\
& b=<\bar{\psi}(0) g G_{\mu \nu} \sigma_{\mu \nu} \psi(0)>_{0}=m_{0}^{2} a ; \quad m_{0}^{2} \sim 0.8 \quad \mathrm{GeV}^{2}
\end{aligned}
$$

$y$ is the recoil parameter, $\sigma$ is the Borel parameter.

Our parameters are connected with the analogous parameters used in Ref. 8 in the following way. The Borel parameter $T$ used in Ref. 8 is $T=2 \sigma$. The spectral density variables used in our eq. (50) are connected with the dimensionless spectral variables used in eq. (3.14) in Ref. 8 as $s_{1}=z \sigma, s_{2}=z^{\prime} \sigma$. The continuum threshold in that paper $\omega_{c}$ is numerically twice as big as the continuum threshold $\omega_{c}$ used in our paper, since the author uses the spectral density variables $\omega, \omega^{\prime}$ equal to $2 s_{1}, 2 s_{2}$ respectively.

Recall that in the nonrelativistic case we make the Borel transform over the light quark virtual energy and $\sigma$ has the dimension of mass, not of mass square as in the relativistic case. This makes the sum rules very similar to the sum rules for the harmonic oscillator considered in the previous section. The area of the integration of the spectral density is denoted by $\Sigma$ and is depicted in Fig. 5. Finally, $E_{R}$ is the mass difference between the mass of the heavy quark and that of the heavy meson (both tend to infinity in our limit). The constant $\mathrm{F}$ is the heavy meson leptonic decay constant:

$$
<0\left|\bar{q} \gamma_{\mu} \gamma_{5} h_{Q}\right| P>=\frac{i F}{\sqrt{2}} v_{\mu}
$$

The field $h_{Q}$ are heavy quarks fields in Heavy Quark Effective Theory ${ }^{4}$. The constant $F$ is related to more familiar $f_{P}$ by $F=f_{P} \sqrt{m_{Q}}$ modulo logs of the type discussed in Ref. 26 .

Although the sum rule is the same, our analysis will be quite different from the analysis in Ref. $7,8,9$, however. In particular we shall argue that the uncertainty in $\rho^{2}$ is quite small, not gigantic as in the latter papers. Indeed, the main unsolved problem in the analysis of the sum rule (50) is the choice of continuum. We saw in the previous section that the considerations due to duality and relevant symmetry severely constrain the possible choice of continuum model in the sum rules. The analysis in the previous section can be exactly 
repeated for the nonrelativistic sum rules in QCD. As a result the only possible choice of the integration area is that depicted in Fig. 5. For every fixed value of $\left(s_{1}+s_{2}\right) / 2$ we have to integrate over all possible values of $A=\left(s_{1}-s_{2}\right)$. Any other prescription for integration of spectral density will be incompatible with the general requirements of duality and angular isotropy ( $\mathrm{S}$ wave states). The variable $\left(s_{1}+s_{2}\right) / 2$ plays the same role as $\mathcal{E}$ in the previous section.

We have argued in a very similar kinematics that duality in the three point functions must be understood in the generalized sense. Namely, the spectral density for the interacting case integrated in the A direction matches that for the free particle only after integration in the A direction. The same kind of generalized duality must also hold for the QCD sum rules.

The generalized duality means that we have to write the sum rule for the spectral density already integrated in the A direction (recall that this is the direction orthogonal to the main diagonal). This effective spectral density will be dependent only on the variable $\left(s_{1}+s_{2}\right) / 2$. Only after this integration we can speak about duality and make a choice of continuum model. We now have the only one possible choice of the continuum model. The continuum is approximated by the spectral density due to the bare loop, integrated over the variable $A=\left(s_{1}-s_{2}\right)$. This is exactly the same continuum model as the model that was used in the two-point sum rules. Numerically, of course, it does not matter whether we assume local duality and take the bare loop contribution integrated over a two-dimensional area depicted on Fig.5 as the continuum model or if we approximate the A integrated continuum contribution by the bare spectral density, integrated in the A direction, starting from some threshold. However our experience with the harmonic oscillator shows, that only the second prescription is physically correct.

We can now write a sum rule for the three point function based only on the generalized duality discussed above. It has the form:

$$
\frac{3}{(y+1)^{2} \pi^{2}} \int_{0}^{\omega_{c}} s^{2} e^{-s / \sigma} d s-\frac{a}{4}+\frac{b(2 y+1)}{192 \sigma^{2}}=\frac{F^{2} \xi(y) \exp -E_{R} / \sigma}{4} .
$$

Here $s=\left(s_{1}+s_{2}\right) / 2$. This sum rule coincides with one of many possible forms of the sum rules (due to different choices of the integration domain $\Sigma$ in eq. (50)) considered in Ref. 8. Now we know that eq. (53) is the only possible form of the sum rule. We also write the sum rule for the slope parameter $\rho^{2}$ directly:

$$
\left(+\frac{3}{4 \pi^{2}} \int_{0}^{\omega_{c}} s^{2} e^{-s / \sigma} d s-\frac{b}{96 \sigma^{2}}\right) \frac{\exp E_{R} / \sigma}{F^{2} / 4}=\rho^{2} .
$$


Consider now the single Borel sum rule. This sum rule was found to work as well as the double Borel sum rule in the case of the harmonic oscillator. We test this sum rule in the heavy quark case.

Recall the basic idea behind this approach. We write the dispersion relation for the polarization operator

$$
\Pi\left(E_{1}, E_{2}, y\right)=\int_{0}^{\infty} \frac{\operatorname{Im} \Pi\left(s_{1}, s_{2}, y\right)}{\left(s_{1}+E_{1}\right)\left(s_{2}+E_{2}\right)} d s
$$

where

$$
\left(v_{1}^{\mu}+v_{2}^{\mu}\right) \Pi\left(E_{1}, E_{2}, y\right)=\int d^{4} x d^{4} y \exp i\left(k_{1} x+k z\right)<j_{5}(x) j_{\mu}(z) j_{5}^{+}(0)>.
$$

In eq. (56) $k_{1}$ is the momentum of the initial heavy meson, $k$ is the transferred momentum, $k_{2}=k_{1}+k$ is the momentum of the final heavy meson; $k_{\mu}=\left(m_{Q}+E_{1}\right) v_{1 \mu}$ and $k_{2 \mu}=$ $\left(m_{Q}+E_{2}\right) v_{2 \mu}$. The current $j_{5}=\bar{Q} \gamma_{5} q$ is the quark current that creates heavy mesons, and $j_{\mu}=\bar{Q} \gamma_{\mu} Q$ is a vector current. Q and $q$ are the heavy and light quark fields respectively. In the double Borel transformed sum rules we make the double Borel transformation of the sum rule (55) with respect to the the variables $E_{1}$ and $E_{2}$ independently and saturate the r.h.s. of eq. (55) by the resonance contributions. In the single Borel transformed sum rules we put $E=E_{1}=E_{2}$ from the very beginning in eq. (55) and then carry out the Borel transformation over the variable E. As it was explained in the previous section in the example of the harmonic oscillator the difference between the double and single Borel transformed sum rules is that we take the contribution of nondiagonal transitions with different weights. The spectral density for the bare loop now depends only on one variable $E$. As it was explained above when we use such spectral density we do it after an implicit integration in the A direction. Thus, the two sum rules are very similar in spirit. The single Borel transformed sum rule has the form:

$$
\Pi\left(x, y, \omega_{c}\right)=\frac{\xi(y) F^{2} \exp \left(-E_{R} / \sigma\right)}{4}+a(y) \sigma .
$$

Here $a(y)$ measures the contribution of nondiagonal transitions. One can easily calculate $\Pi$ in this case taking into account the same operators as we took into account writing the double Borel sum rule. The result is

$$
\begin{aligned}
& \frac{3}{4 \pi^{2}} \frac{1}{y+1}\left(1+\frac{1}{\sqrt{y^{2}-1}} \log \left(y+\sqrt{y^{2}-1}\right)\right) \int_{0}^{\omega_{c}} s^{2} e^{-s / \sigma} d s \\
& -a / 4+\frac{b(4 y+5)}{576 \sigma^{2}}=\frac{F^{2}}{4}(\xi(y)+a(y) \sigma) \exp \left(-E_{R} / \sigma\right)
\end{aligned}
$$




\section{ANALYSIS OF THE SUM RULES.}

Let us now analyse our sum rules. We start from the sum rule (53). In order to minimize the effects related to the uncertainty in $F, E_{R}$ we deal with the sum rule (53) divided by the sum rule for $F, E_{R}$ (Ref. $\left.1,7,8,9\right)$ :

$$
\xi(y)=\frac{I\left(\sigma, y, \omega_{c}\right)}{I_{M}\left(\sigma, \omega_{c}\right)} \equiv G\left(\sigma, y, \omega_{c}\right) .
$$

Here

$$
I\left(\sigma, y, w_{c}\right)=\frac{3}{(y+1)^{2} \pi^{2}} \int_{0}^{\omega_{c}} s^{2} e^{-s / \sigma} d s-\frac{a}{4}+\frac{b(2 y+1)}{192 \sigma^{2}}
$$

and

$$
I_{M}\left(\sigma, \omega_{c}\right)=\frac{3}{4 \pi^{2}} \int_{0}^{\omega_{c}} s^{2} e^{-s / \sigma} d s-\frac{a}{4}+\frac{b}{64 \sigma^{2}} .
$$

The function $I_{M}\left(\sigma, \omega_{c}\right)$ is the theoretical part of the sum rule for the mass and leptonic decay constant of heavy meson.

$$
I_{M}\left(\sigma, \omega_{c}\right)=\frac{F^{2}}{4} \exp \left(-E_{R} / \sigma\right)
$$

The continuum threshold is chosen to be the same as for the two-point sum rules. We did not find any significant dependence of the continuum threshold on the recoil $y$. We depict the behaviour of $\xi(y)$ for different values of the continuum threshold in Fig.6. We find only a small uncertainty $\sim 10 \%$ due to the choice of the continuum threshold. Let us also remind once again that we assume that the generalized duality picture is valid. Hence our continuum model looks like the continuum model for the sum rules for the two point function and has a single threshold $\omega_{c}$. We vary this threshold to obtain the best stability of the sum rules.

Let us now proceed to the sum rules for $\rho^{2}$, eq. (54). The dependence of the r.h.s. of this sum rule on $\sigma$ is depicted in Fig.7. We immediately get:

$$
\rho^{2}=0.7 \pm 0.1
$$

Consider now the single Borel transformed sum rules. We rewrite this sum rule in the form:

$$
a(y) \sigma+\xi(y)=\frac{I\left(\sigma, y, \omega_{c}\right)}{I_{M}\left(\sigma, \omega_{c}\right)} .
$$

Here the function $I$ is the l.h.s. of the sum rule (58). The function $a(y)$ is the $x$-independent function that measures the contribution of the nondiagonal transitions. The l.h.s. of the 
sum rule (64) is well approximated by a straight line for $\sigma \geq 0.8 \mathrm{GeV}$. The corresponding behaviour of the Isgur-Wise function is depicted in Fig.8. Notice that the sum rule (60) gives slightly smaller $(\sim 10 \%$ at $y=2)$ values of $\xi(y)$ than the sum rule (54) . Nevertheless, the difference between the predictions of these two sum rules is inside error bars for each of them.

We can now differentiate the sum rule (60) with respect to $y$ at $y=1$. Then we get the sum rule for $\rho^{2}$. This sum rule gives the value

$$
\rho^{2}=0.55 \pm 0.1
$$

Thus we get a slightly smaller value of $\rho^{2}$ using the sum rule (60). The difference is, however, within the error bars.

It is tempting to speculate that the true value of $\rho^{2}$ lies in the intersection of the predictions of the single and double Borel sum rules. After all, these two sum rules worked equally well in the case of the harmonic oscillator. This gives $\rho^{2} \sim 0.6-0.65$.

For more careful estimate, we take the average of the predictions of these two some rules. We have

$$
\rho^{2}=0.65 \pm 0.15
$$

as the average from the predictions of these two sum rules.

Let us now turn to the check of self-consistency of our approach. The nontrivial check is provided by comparison of our results with the predictions of the Bjorken sum rule ${ }^{15}$.

The Bjorken sum rule ${ }^{15}$ has the form

$$
\rho^{2}=\frac{1}{4}+\sum_{m=1}^{m_{\max }}\left|\tau_{1 / 2}^{m}(1)\right|^{2}+2 \sum_{m=1}^{p_{\max }}\left|\tau_{3 / 2}^{m}(1)\right|^{2}+\ldots
$$

Here $\tau_{1 / 2}(1), \tau_{3 / 2}(1)$ are nondiagonal transition formfactors from the multiplet of pseudoscalar and vector mesons into multiplets with $s_{l}^{\pi_{l}}=1 / 2^{+}$and $3 / 2^{+}$respectively taken at zero recoil. Here $s_{l}$ and $\pi_{l}$ are the spin and parity of the light quark in the meson. These formfactors are defined in the following way:

$$
\begin{gathered}
\left.\frac{<A\left|A_{\mu}\right| P>}{m}\right|_{y=1}=-4 \tau_{1 / 2}\left(v_{1}-v_{2}\right)_{\mu} \\
\left.\frac{<B\left|A_{\mu}\right| P>}{m}\right|_{y=1}=-\frac{1}{\sqrt{2}} i \tau_{3 / 2} \epsilon_{\mu \alpha \beta \gamma} \epsilon^{* \alpha}\left(v_{1}+v_{2}\right)^{\beta}\left(v_{1}-v_{2}\right)^{\gamma}
\end{gathered}
$$


Here $A$ is the scalar heavy meson in $1 / 2^{+}$multiplet, and $B$ denotes the state with $J^{P}=1^{+}$ in $3 / 2^{+}$multiplet of heavy mesons. We use the notations of Ref. 14,16. For general values of recoil $y$ the formfactors $\tau_{1 / 2}, \tau_{3 / 2}$ are new universal functions of the recoil analogous to the Isgur-Wise function. All other formfactors for transitions between $1 / 2^{-}$and $1 / 2^{+}, 3 / 2^{+}$ multiplets can be expressed through $\tau_{1 / 2}$ and $\tau_{3 / 2}$. ${ }^{16}$

It is possible to analyse the formfactor $\tau_{1 / 2}(1)$ using the QCD sum rules in which we do double Borel transformation. Taking into account only the contribution of the unit operator and the quark condensate we obtain the following sum rule for $\tau_{1 / 2}$ :

$$
\begin{aligned}
& \lim _{y \rightarrow 1} \frac{3}{4 \pi^{2} \sqrt{y^{2}-1}(y-1)} \int_{\Sigma}\left(s_{1}-s_{2}\right) \exp \left(-s_{1} / \sigma_{1}-s_{2} / \sigma_{2}\right) d s_{1} d s_{2}- \\
& a / 2=8 \tau_{1 / 2} \frac{\lambda_{S} \lambda_{P}}{4} \exp \left(-E_{S} / \sigma_{1}-E_{P} / \sigma_{2}\right)
\end{aligned}
$$

A rough estimate using this sum rule gives

$$
\tau_{1 / 2}(1) \sim 0.35 \pm 0.2
$$

Note very high uncertainty in this estimate. First, we took into account only two operators of dimensions zero (unit operator) and 3 (light quark condensate). Second, and more important, we did not take into account continuum contribution. In eq. (70) $\lambda$ is a semileptonic decay constant of the corresponding resonance:

$$
<0|j| S(P)>=\lambda_{S(P)} \phi_{S(P)}
$$

where $j$ is the quark current that creates a meson, $\bar{Q} q$ for scalar meson $\mathrm{S}$ and $\bar{Q} \gamma_{5} q$ for pseudoscalar meson $\mathrm{P}, \phi$ is the corresponding wave function.

It is interesting to note that the same ratio $\tau_{1 / 2} / \xi(1)$ can be obtained by combining nonrelativistic quark model and predictions obtained using the nonrelativistic sum rules for the two-point functions. ${ }^{1}$ Indeed, in the nonrelativistic quark model ${ }^{16}$

$$
\tau_{1 / 2}(1) \sim \int d^{3} k \phi_{11}^{*}(k) k \phi_{00}(k)
$$

Here $\phi_{11}$ is the radial wave function of the scalar resonance, and $\phi_{00}$-of pseudoscalar meson. For the rough estimate we have

$$
\tau_{1 / 2}(1)=\frac{\tau_{1 / 2}(1)}{\xi(1)}=\frac{\left|\phi_{11}(0)\right|}{\left|\phi_{00}(0)\right|}=\sqrt{\frac{\lambda_{P}}{\lambda_{S}}} \sim 0.3
$$


The latter equation follows from ${ }^{1}$

$$
\lambda^{2}=12|\phi(0)|^{2}
$$

The determination of $\tau_{3 / 2}$ is much more cumbersome since the corresponding currents contain derivatives. However, following the nonrelativistic quark model we can accept for orientation that $\tau_{3 / 2} \sim \tau_{1 / 2}$. Note also that the contribution of the radially excited states to Bjorken sum rule is strongly suppressed (it is zero in nonrelativistic quark model). We now take for $\tau_{1 / 2}$ the value (see eqs. (71),(74)) $\tau_{1 / 2}(1) \sim \tau_{3 / 2}(1) \sim 0.35$. Then we get for $\rho^{2}$ an estimate:

$$
\rho^{2} \sim \frac{1}{4}+3 \tau_{1 / 2}(1)^{2} \sim 0.25+3(0.35 \pm 0.2)^{2}
$$

We finally conclude that Bjorken sum rule implies

$$
0.35 \leq \rho^{2} \leq 1.15
$$

This result is in agreement with the sum rule prediction (63) .

\section{CONCLUSION}

We have used the QCD sum rule method to find the shape of the Isgur-Wise function and its slope parameter $\rho^{2}$. We have discussed the nature of the uncertainties pointed out in Ref. 8,9 and shown that they can be eliminated if we implement the general requirements of duality in the appropriate way. We used a toy model of a harmonic oscillator to discuss in detail the consequences of the demand of duality and relevant symmetries for the sum rules for formfactors.

In particular, we have shown on the example of harmonic oscillator that there is no local duality in the sum rules for the three point functions. Duality must be understood in a generalized sense, between integrated quantities. The proper duality severely constrains the form of the continuum model.

For the case of heavy mesons the requirements of duality lead to the double Borel sum rules (53), (54). The corresponding behaviour of the Isgur-Wise function as a function of recoil is depicted in Fig.6. For the slope $\rho^{2}$ we get

$$
\rho^{2}=+0.7 \pm 0.1
$$


The single Borel transformed sum rules lead to the shape of Isgur -Wise function depicted on Fig. 8. They lead to the slightly smaller (within 10\%) value of $\xi(y)$. The difference between the predictions of these two sum rules is within the error bars however. For the slope parameter we obtain

$$
\rho^{2}=0.55 \pm 0.1
$$

For $\rho^{2}$ we can argue that the values (79) and (78) are upper and low bounds on the value of the slope parameter. Taking the average of these two estimates, we have

$$
\rho^{2}=0.65 \pm 0.15
$$

Note that our predictions for the slope parameter lead to the smaller values than those obtained by analysing the phenomenological data: $\rho^{2} \sim 1.4 \pm 0.4$ in Ref. 27 and $\rho^{2} \sim 1.2 \pm 0.4$ in Ref. 28.

On the other hand, our results for the slope parameter are very close to the results of Ref. 14. The model developed in the latter paper gives the value $\rho^{2} \sim 0.7$.

After this work was completed we have learnt about the results for $\rho^{2}$ obtained from the so called optical sum rules ${ }^{29}$ extending the Bjorken approach. ${ }^{15}$ The analysis in Ref. 29 leads to the inequality $\rho^{2} \leq 0.75$. This is in a very good agreement with our estimate for $\rho^{2}$ given by eqs. (78) , (79), (80).

We are grateful for to A. Vainshtein and I. Kogan for useful discussions. 


\section{REFERENCES}

1. E. Shuryak, Nucl. Phys., B198 (1982) 83.

2. N. Isgur and M. Wise, Phys. Lett., B232 (1989) 113; B237 (1990) 527.

3. M. Voloshin and M. Shifman, Yad. Fiz., 47(1988) 801 [Sov. J. of Nucl. Phys., 47 (1988) $511]$.

4. E. Eichten and B. Hill, Phys. Lett., B234 (1990) 511

H. Georgi, Phys. Lett., B240 (1990)447.

5. M. Wise, New symmetries of strong interactions, preprint CALT 68-1721, H. Georgi, Heavy quark effective field theory, preprint HUPT-91-A039, 1991;

B. Grinstein, Light-Quark, Heavy-Quark systems, preprint SSCL-34,1992 (to be published in Ann. Rev. Nucl. Part. Sci.).

6. M. Neubert, V. Rieckert, B. Stech and Q.P. Xu , Heidelberg preprint HD-THEP -91-28 (1991).

7. A. Radyushkin, Phys. Lett.,B271 (1991) 218.

8. M. Neubert, Phys. Rev., D45 (1991) 2451 .

9. P. Ball, Phys. Lett., B281 (1992) 133.

10. M. Shifman, A. Vainshtein and V. Zakharov, Nucl. Phys., B147 (1979) 385, 448.

11. B. L. Ioffe and A. V. Smilga, Phys. Lett., B114 (1982) 353.

12. A. V. Nesterenko and A. V. Radyushkin, Phys. Lett., B115 (1982) 410.

13. B. L. Ioffe and A. V. Smilga, JETP Lett., 37 (1983) 298. 
14. N. Isgur, D. Scora, B. Grinstein, M. Wise, Phys. Rev., D39 (1989) 799;

B. Grinstein, M. B. Wise and N. Isgur, Phys. Rev. Lett., 56 (1986) 258.

15. J.D. Bjorken, preprint SLAC-PUB-5278 (1990), Invited talk at Les Recontre de la valle d'Aosta, La Thuille, Italy, (1990).

16. N. Isgur and M. Wise, Phys. Rev., D43 (1991) 819.

17. S. Flugge, "Practical Quantum Mechanics", Springer-Verlag, 1965.

18. R. Feynman and A. Hibbs, "Quantum Mechanics and Path Integrals" McGraw-Hill, 1965.

19. M. Shifman, Ann. Rev. Nucl. Part. Sci., 33 (1983) 199.

20. J. Bell and R. Bertlmann, Nucl. Phys., B177 (1981) 218;

A. I. Vainshtein et al, Yad. Fiz.,32 (1980) 1622 [Sov. J. of Nucl. Phys., 32 (1980) 840].

21. B. V. Geshkenbein and M. S. Marinov, Yad. Fiz., 30 (1979) 1400 [Sov. J. of Nucl. Phys., 30 (1979) 726 ].

22. V.A. Nesterenko and A. I. Radyushkin, JETP Lett., 39 (1984) 707.

23. M. Beilin and A.I. Radyushkin, Int. J. of Mod. Phys., A3 (1988) 1183.

24. I.I. Balitsky and A. Yung, Phys. Lett., 129B (1983) 388.

25. V. Eletsky and I. Kogan, Z. Phys., C28 (1985) 155.

26. M. Voloshin and M. Shifman, Yad. Fiz.,45 (1987) 463 [Sov. J. Nucl. Phys., 45 (1987) $292]$.

27. J.L. Rosner, Phys. Rev., D42 (1990) 3732.

28. M. Neubert, Phys. Lett.,B264 (1991) 455. 
29. M. Voloshin, preprint TPI-MINN-92/25-T (May 1992). 


\section{Figure Captions}

Fig.1: Heavy quark in Breit reference frame a) before and b) after the emission .

Fig.2: The spectral density for $\rho_{\text {n.r. }}^{2}$ for the oscillator. The difference between $\bullet$ and 0 is the sign of the residue.

Fig.3: Boundaries of the domain where the spectral density $\sigma_{\text {bare }} \neq 0$.

Fig.4: The only possible geometry of the duality domains.

Fig.5: The duality domain for the sum rule for Isgur-Wise function.

Fig.6: The Isgur-Wise function $\xi(y)$.

Fig.7 The dependence of the l.h.s. of the sum rule for $\rho^{2}$ on the Borel parameter $x$.

Fig. 8 The Isgur-Wise function from the single Borel transformed sum rule. 